\title{
FENOLOGÍA Y VOLUMEN DE FLORACIÓN DEL MATORRAL DE LA SIERRA DE MIJAS (MÁLAGA, S. ESPAÑA)
}

\author{
M. Isabel HIDALGO y Baltasar CABEZUDO
}

\begin{abstract}
RESUMEN. Fenología y volumen de floración del matorral de la Sierra de Mijas (Málaga, S. España). Se ha realizado un estudio sobre la variación anual del volumen de floración total (VFT) en un matorral del Sur de España (Málaga). Para ello hemos tenido en cuenta la fenología y cobertura de 237 especies. VFT y floración presentan ritmos típicamente mediterráneos, con máximos primaverales (abril) y mínimas estivales (septiembre), siendo numerosas las especies con largos periodos de floración total y en pico. La existencia de niveles adecuados de VFT durante todo el año permite el aprovechamiento apícola continuado del matorral de la zona.
\end{abstract}

Palabras clave. Melitopalinología, fenología, floración, S. España, Málaga

ABSTRACT. Flowering quantification and phenology in the schrublans of Sierra de Mijas (Malaga, S. Spain). A study about the annual variation of the "total flowering volume" (TFV) for a schrubland comunity was carried out in Malaga (Spain). For this study we have taken into account the phenology and the vegetal cover of 237 species. The TFV and flowering volume and its secuence show tipically mediterranean rythmics, where the seasonal periods are very marked, with maximum values in Spring (April) and minimum values in Summer (September). However, many species present long flowering periods, which allows a continuous development of the beekeeping in this area.

Key words. Melissopalynology, flowering phenology, schrublands, S. Spain, Malaga.

\section{INTRODUCCIÓN}

Los estudios de potencialidad apícola son de gran interés para poder conocer y mejorar los recursos melitopalinológicos de un territorio. La productividad de una colmena depende, además de sus características intrínsecas, de una serie de factores como vegetación, flora, fenología de la floración, abundancia de determinadas especies y tipo de clima.

La importancia de los estudios sobre fenología floral en trabajos de melitopalinología, se basa en la estrecha relación que existe entre polinizadores, periodo de floración y épocas de aprovechamiento (Saury,1981). El interés que en estos últimos años se ha despertado por el estudio de la dinámica de las comunidades vegetales, ha provocado un incremento en trabajos fenológicos aplicables a los estudios de biología floral, melitopalinología, conservación etc. (Martín y Escarre, 1980; Arroyo, 1983, 1985, 
1988 a y b; Herrera, 1985 b, 1986, 1987 a y b, 1988 a y b; Ortiz, 1991; Cabezudo et al., 1993, 1992; Navarro et al., 1993).

La participación que puede tener una especie en el volumen total de floración (VFT) de un territorio y en una época determinada, va a depender no sólo de su fenología sino de la cobertura que cada una de ellas tenga en dicho territorio (Herrera, 1985a); una sola especie con gran cobertura puede aportar la suficiente cantidad de polen o néctar que permita la actividad de una colmena. La cobertura vegetal de las especies del área de estudio es un dato que, unido a la cuantificación de otros factores (recompensas florales, fenología, producción floral), va a permitir estimar la importancia melífera y polinífera de dichas especies en la zona.

Nuestro trabajo se ha centrado en la Sierra de Mijas (Málaga), y ha tenido como objetivo principal la evaluación semanal del volumen total de floración a lo largo de un año, por lo que hemos tenido que hacer previamente un estudio sobre cobertura y fenología de las especies mas importantes de la zona.

La Sierra de Mijas se encuentra situada en la provincia de Málaga (España), al SW de la capital. Comprende parte de los términos municipales de Mijas, Alhaurín el Grande, Alhaurín de la Torre y Benalmádena. Climáticamente se encuadra en la región Mediterránea (fig. 1), con épocas de lluvia corta en otoño-invierno y máxima insolación en verano. El periodo de mayor aridez se sitúa desde finales de Mayo hasta finales de Septiembre. Desde un punto de vista biogeográfico la zona estudiada pertenece a la Región Mediterránea, y en función de sus peculiaridades florísticas y fitosociológicas (vegetación) se incluye, junto con territorios próximos, en el Subsector Mijense del Sector Rondeño (Nieto et al., 1991). La similitud florística que presenta todo el sector hace que, salvo variaciones de tipo altitudinal, gran parte de las conclusiones melitopalinológicas y fenológicas referentes

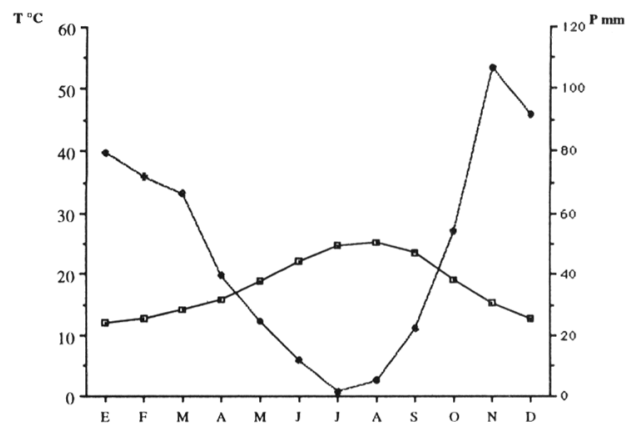

Figura 1. Diagrama ombrotérmico (30años) de la Sierra de Mijas (Málaga). Hydrothermic diagram (30 years) of Sierra de Mijas (Málaga).

al territorio estudiado puedan aplicarse a todo el sector.

La vegetación del territorio estudiado (Subsector Mijense) está representada por un coscojar-encinar perteneciente a la asociación Rhamno velutini-Quercetum cocciferae (Nieto et al., 1987-88), formada por bosquetes de fanerófitos y nanofanerófitos que colonizan suelos sobre mármoles dolomíticos. El jaralaulagar, predominante en la Sierra de Mijas, pertenece a la asociación Cisto clussiUlicetum baetici (Nieto et al., 1987-88) y aparece por degradación del encinar-coscojar, producto de la humanización del territorio (ganado, fuego, cortes), o más generalmente por el escaso desarrollo edáfico de gran parte del territorio(afloramientos pedregosos, fuertes pendientes, etc.). Otros tipos de comunidades muy importantes florísticamente son las que, con carácter pionero, se instalan sobre arenas dolomíticas, constituidas por hemicriptófitos y terófitos efímeros del Sector Rondeño.

El estado actual de la cubierta vegetal de la sierra es una consecuencia directa de los usos tradicionales y actuales; alguno de ellos conservativos (apicultura, plantas medicinales y aromáticas), pero la mayoría altamente degradativos (canteras, urbanizaciones, etc.). La apicultura es un aprovechamiento tradicional de la zona, encontrándose en la actualidad numerosos asentamientos de colmenas, sobre 
todo en épocas invernales y primaverales. La tendencia de los últimos años a la repoblación forestal con coníferas (pinos) ha afectado a una gran superficie de la Sierra de Mijas, modificando el paisaje vegetal de la misma, no sólo por la predominancia de estas especies sino por el deterioro del suelo que han producido prácticas silviculturales poco adecuadas.

\section{MATERIAL Y MÉTODOS}

Para la elección de las parcelas utilizadas en los estudios fenológicos y de cobertura, se ha tenido en cuenta principalmente la diversidad florística, estado de conservación y accesibilidad. Para la estimación de la cobertura de las especies leñosas se ha elegido el método «Line Intercept» desarrollado por Canfield (1941). El número de transectos empleados ha sido de 65 , teniendo cada uno de ellos una longitud de 50 metros, siendo la longitud total analizada de 3250 metros.

Para la estimación de la cobertura de las especies herbáceas de interés apícola, el método utilizado ha consistido en establecer diez parcelas de $4 \mathrm{~m}^{2}$ en la zona de herbazales, en cada una de las cuales se ha contabilizado el número de individuos presentes, así como el porcentaje de aparición de las especies en las distintas parcelas. El muestreo se ha llevado a cabo en el periodo final de invierno y al final de la primavera, con objeto de cubrir dos fechas en las que aparecen ciertas herbáceas de interés apícola que no estaban en el momento de las estimación de la cobertura general.

Se ha estudiado la fenología floral de la mayoría de los caméfitos y fanerófitos autóctonos presentes en la zona, y de aquellas otras especies (terófitos, geófitos, hemicriptófitos y cultivadas) que por su abundancia y/o interés melífero puedan ser relevantes. Para la elección de dichas especies hemos tenido en cuenta los datos aportados para la zona por Pérez Sanz et al. (1987) y los referentes a flora melífera aportados por diversos autores (Herce,1942; Howes, 1953; Rabiet, 1977; Ricciardelli y Persano, 1981; Rita, 1983; Bonet et al., 1985; Socorro, 1985; Ortega, 1987; Ortiz et al., 1990; Ortiz, 1985, 1988 y 1990 a y b; Talavera et al., 1988; Ortiz y Fernández, 1992; Serra, 1988; Hidalgo et al., 1990).

El seguimiento de la fenología se ha realizado durante tres años, y dada la similitud de los resultados, se han utilizado para las gráficas únicamente los datos del periodo Febrero 1988/Febrero 1989, periodo que se corresponde con el de toma de muestras de las colmenas y medidas de néctar y polen .

La metodología empleada ha sido básicamente la propuesta por Anderson y Hubricht (1940) y Herrera (1985a), basada en el efecto visual que causan en el paisaje la floración de los distintos taxones. Se han establecido seis fases de floración: 0, floración ausente; 1, inicio de floración (desde la primera flor observada hasta aproximadamente el $10 \%$ de la floración máxima); 1-3, floración ascendente (del 10 al 50\% de la floración máxima); 3 , floración máxima (del 50 al 100\% de floración); 3-2, floración descendente (del 50 al 10\% de la floración máxima) y 2 , floración terminal (menos del $10 \%$ de la floración máxima). Para la representación gráfica de los fenogramas se han transformado las categorías anteriores en los siguientes valores absolutos: 1 y $2=1$ (línea de puntos); 1-3 y 3-2 = 2 (línea continua fina); $\mathbf{3}=3$ (línea continua gruesa) y $\mathbf{0}=0$ (en blanco). Las categorías $\mathbf{1 - 3}, \mathbf{3}$ y 3-2 se corresponden con el periodo fenológico que denominamos "pico de floración» (cuando al menos el $10 \%$ de los individuos de una especie se encuentra en floración).

Las observaciones se han efectuado en unos itinerarios fijos situados en las zonas mas características de la sierra, y con longitud variable en función de permitirnos la observación de una amplia diversidad de especies (Hilty,1980). Cada fase se ha estimado sobre 


\begin{tabular}{ll|ll}
\hline Especies & M\% & Especies & M\% \\
\hline Rosmarinus officinalis & 8.62 & Helianthemum croceum & 0.54 \\
Ulex baeticus & 7.69 & Anthyllis cytisoides & 0.48 \\
Cistus albidus & 6.02 & Teucrium fruticans & 0.46 \\
Pinus halepensis & 5.81 & Thapsia villosa & 0.43 \\
Cistus clusii & 3.59 & Andryala ramossisima & 0.40 \\
Thymbra capitata & 3.18 & Asphodelus albus & 0.33 \\
Juniperus oxycedrus & 2.94 & Echium albicans & 0.32 \\
Quercus rotundifolia & 2.77 & Calicotome villosa & 0.3 \\
Stipa tenacissima & 2.64 & Carlina corymbosa & 0.25 \\
Quercus coccifera & 1.76 & Helichrysum stoechas & 0.25 \\
Cistus monspeliensis & 1.42 & Ephedra fragilis & 0.23 \\
Chamaerops humilis & 1.41 & Thymelaea hirsuta & 0.23 \\
Phlomis purpurea & 1.36 & Ptilostemum hispanicum & 0.21 \\
Lavandula stoechas & 1.3 & Rhamnus myrtifolius & 0.21 \\
Brachypodium retusum & 0.94 & Daphne gnidium & 0.20 \\
Rhamnus velutinus & 0.90 & Cephalaria leucantha & 0.16 \\
Pistacia lentiscus & 0.78 & Fumana thymifolia & 0.15 \\
Thymus mastichina & 0.75 & Helianthemum lavandulifolium & 0.13 \\
Teucrium lusitanicum & 0.61 & Linum suffruticosum & 0.1 \\
Helianthemum croceum & 0.54 & Satureja graeca & 0.06 \\
\hline
\end{tabular}

Tabla 1. Porcentaje de cobertura lineal de las especies más abundantes de la Sierra de Mijas. Lineal cover percentage of the most abundant species in Sierra de Mijas (Málaga).

el conjunto de plantas de una misma especie, por lo que los porcentajes aproximados se refieren al momento en que se encuentran la mayoría de los ejemplares observados respecto al máximo de floración; la periodicidad de las observaciones ha sido semanal (Herrera,1986; Ortiz,1991).

El "volumen de floración total" (VFT) nos da una idea indirecta de la cantidad de recursos (polen y néctar) que hay en cada periodo del año, y depende fundamentalmente de la fenología y cobertura de cada especie (Herrera 1985a); viene definido por la formula $\mathbf{V F T}=\Sigma \mathbf{V F}_{\mathrm{i}, \mathrm{t}}$; donde $\mathbf{V F}_{\mathrm{i}, \mathrm{t}}$ es el volumen de floración de la especie i en el momento t, que a su vez viene dado por la fórmula $\mathbf{V F} \mathbf{F}_{i, t}=\mathbf{A}_{i} \mathbf{x}$ $\mathbf{I}_{\mathrm{i}, \mathrm{t}}$; donde $\mathbf{A}_{\mathrm{i}}$ es la abundancia relativa de la especie $\mathbf{i}$ en el territorio y $\mathbf{I}_{\mathrm{i}, \mathrm{t}}$ la intensidad de la floración de la especie i en el momento $\mathbf{t}$ (semana).
Para cada semana del periodo de estudio, se ha calculado la intensidad de floración de cada especie (1, 2 y 3 ), multiplicado por la abundancia de la misma expresada en tantos por ciento de cobertura lineal para aquellas especies con valores mayores al $1 \%$ de cobertura; a las especies con valores inferiores a $1 \%$ se les ha asignado el valor 1 de cobertura.

\section{RESULTADOS Y CONCLUSIONES}

Las especies mas abundantes de la sierra, en función de su cobertura lineal (en porcentaje sobre los $3200 \mathrm{~m}$ inventariados), se indican en la tabla 1. La cobertura lineal de todas las especies estudiadas representa el 65,4\% (2092 metros) de la superficie inventariada, destacando como especies mas representativas 


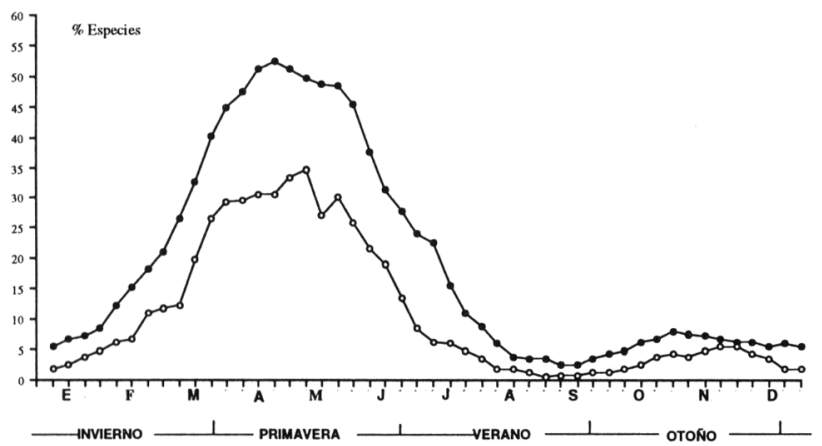

Figura 2. Porcentaje de especies en flor (๑) y en pico de floración (o). (Número de especies estudiadas 237). Percentage of species showing flowering (•) and percentage of species in full blomm (o); (over 237 studied species).

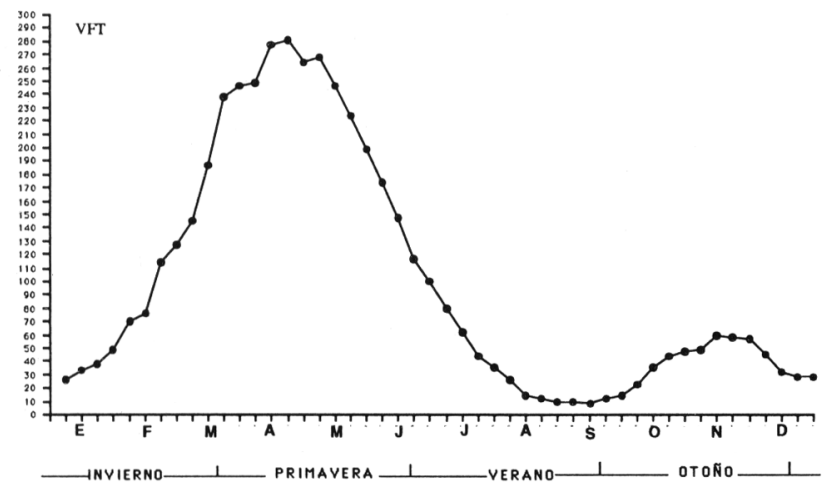

Figura 3. Volumen de Floración Total (VFT) de la Sierra de Mijas en función del periodo fenológico y porcentaje de cobertura de cada especie. Total flowering volume (TFV) in Sierra de Mijas in relation to seasonal periods and each species coverage.

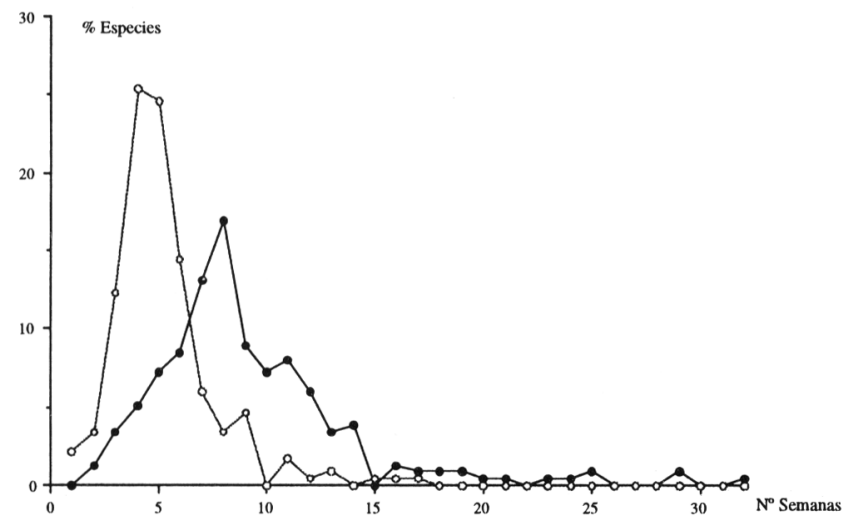

Figura 4. Duración en semanas de los periodos de floración total (•) y pico de floración (o) de las especies estudiadas. Duration (in weeks) of the blooming periods (•) as well as the blooming peaks (o) for the studied species. 
Rosmarinus officinalis, Ulex baeticus, Cistus albidus y Pinus halepensis que en conjunto representan el 28,14\%de la longitud inventariada y el $43,04 \%$ de la cobertura lineal de toda las especies. En la tabla 2 se indican las especies herbáceas mas importantes de la zona en función de su frecuencia de aparición en las 10 parcelas analizadas $(40 \mathrm{~m} 2)$ y del numero total de individuos presentes en dichas parcelas, destacando Galactites tomentosa, Oxalis pes-caprae y Calendula arvensis.

La fenología de floración de las 237 especies estudiadas viene representada en la tabla 3, y están ordenadas a partir de Enero por fechas de inicio de floración. Cada mes está representado por los datos correspondientes a 4 semanas, con un total de 48 semanas. En la figura 2 se representan los porcentajes de especies en periodo de floración y en pico de floración, observándose la existencia de un gran porcentaje de especies de floración primaveral y una fuerte caída de la floración en el periodo veraniego. El máximo porcentaje de especies en floración se sitúa en la última semana de Abril (53\%), y el máximo porcentaje en pico de floración se sitúa en la segunda

\begin{tabular}{lcc}
\hline Especies & F & A \\
\hline Galactites tomentosa & 10 & 754 \\
Oxalis pes-caprae & 9 & 119 \\
Calendula arvensis & 10 & 83 \\
Fedia cornucopiae & 5 & 31 \\
Eruca sativa & 7 & 26 \\
Silene colorata & 5 & 11 \\
Cynoglossum cheirifolium & 4 & 9 \\
Diplotaxis virgata & 6 & 7 \\
Anchusa azurea & 5 & 7 \\
Borago officinalis & 3 & 6 \\
Psoralea bituminosa & 2 & 3 \\
\hline
\end{tabular}

Tabla 2. Frecuencia (F) y abundancia (A) de las especies herbáceas estudiadas. (Número de parcelas inventariadas $=10$ ). Number of plots in which the herbaceus species were found $(F)$ and total number of individuals in these plots (A.). semana de Mayo (44\%). Los valores mínimos de floración total y de pico de floración se dan al final del periodo veraniego (Septiembre). El valor máximo de floración otoñal se produce en la primera semana de Noviembre $(8 \%)$. Los resultados son muy similares a los obtenidos por otros autores en comunidades de zonas mediterráneas próxima (Martín y Escarre, 1980; Arroyo, 1983, 1988 a y b, 1990; Herrera 1985 a, 1986 y Ortiz, 1991), y se corresponden con el tipo de clima característico del territorio estudiado.

Los resultados del "Volumen de Floración Total" (VFT) vienen representados, en periodos semanales, en la figura 3 e indican su variación a lo largo del periodo estudiado, siendo la primavera (abril) el periodo con VFT más alto y el verano (septiembre) el periodo con VFT menor. El volumen de floración otoñal se potencia por la gran cobertura que presentan las escasas especies que florecen en dicha época. Estos resultados concuerdan con los de Herrera (1985a).

La duración (en semanas) de los periodos de floración total y en pico de todas las especies, viene indicado (en porcentaje) en la Gráfica 4. Dicho periodo oscila, para floración total, entre 2 y 32 semanas, con una duración media de 9,16 $\pm 4,64$ semanas, y para el pico de floración entre 1 y 17 semanas, con una media de 5,20 $\pm 2,38$ semanas. La duración mas frecuente de floración total es de 8 semanas (40 especies) y la de pico de floración de 4 semanas (60 especies). Por lo general las especies con floraciones más largas, Rosmarinus officinalis, Calendula arvensis, Diplotaxis virgata, Lobularia maritima, Thymelaea hirsuta y Andryala ragusina, florecen en el periodo del año con menor número de especies en flor (invierno).

AGRADECIMIENTOS. Este trabajo se ha realizado con la ayuda concedida por la Consejería de Educación y Ciencia de la Junta de Andalucía al Grupo de Investigación 4013. 


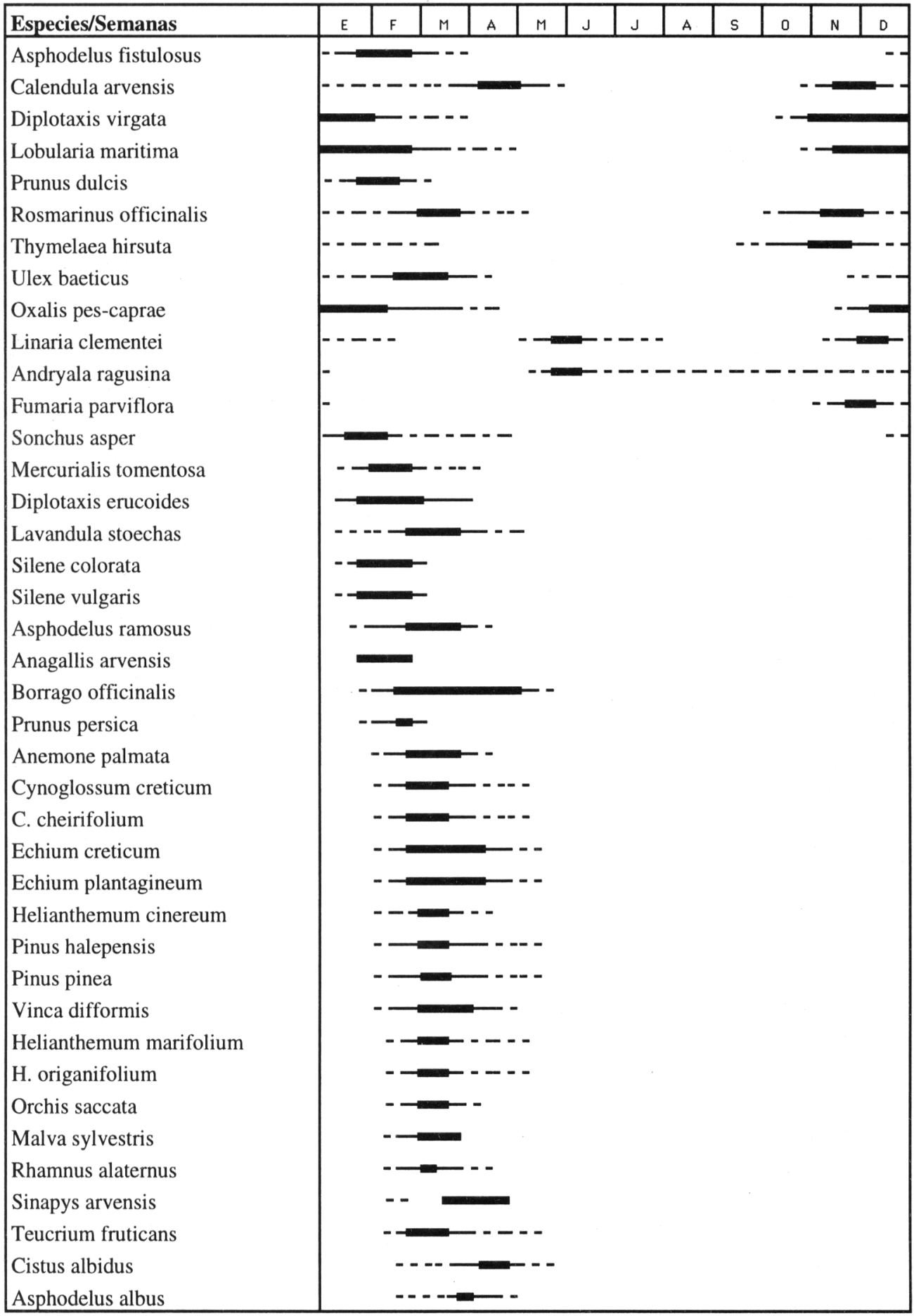




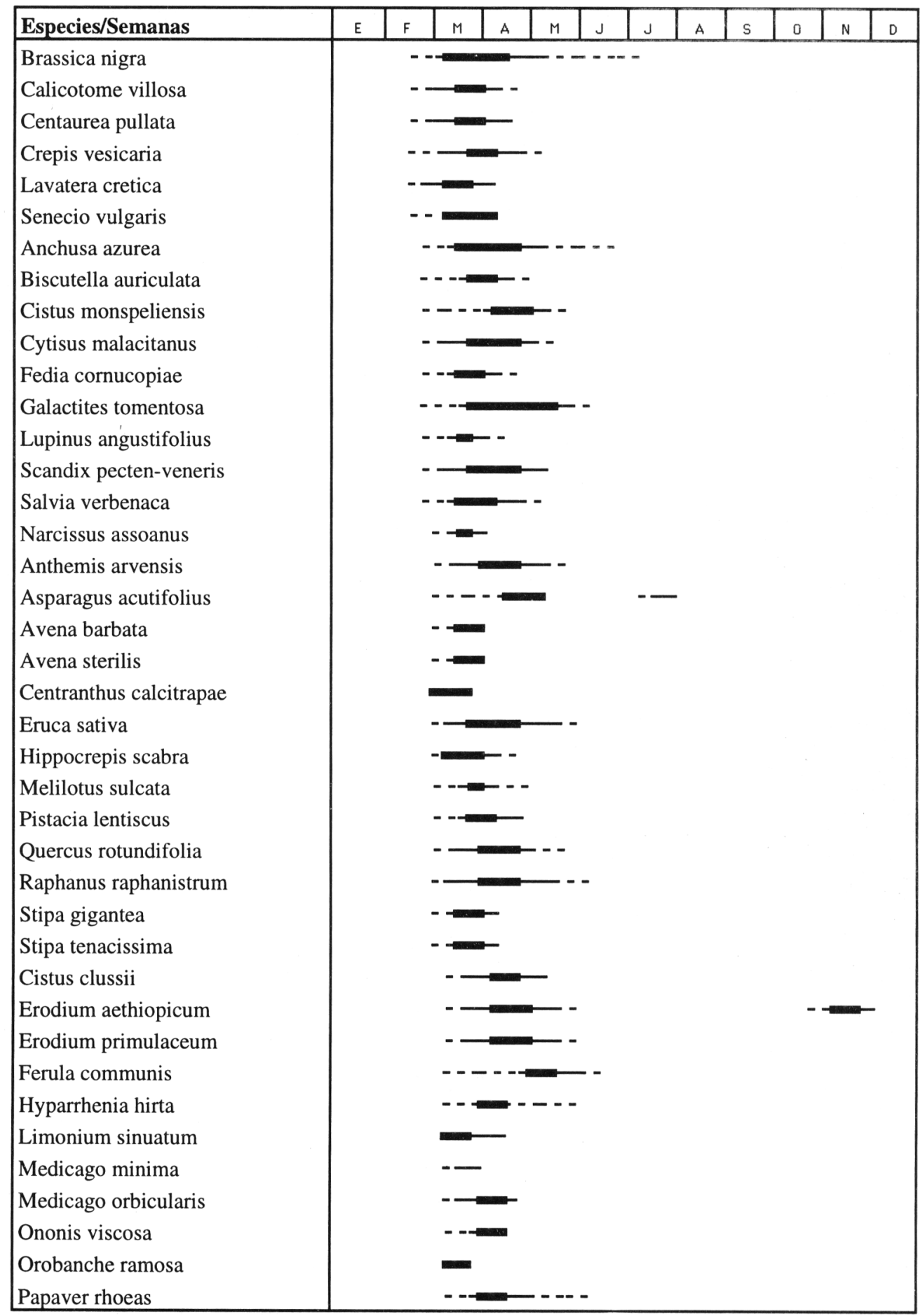




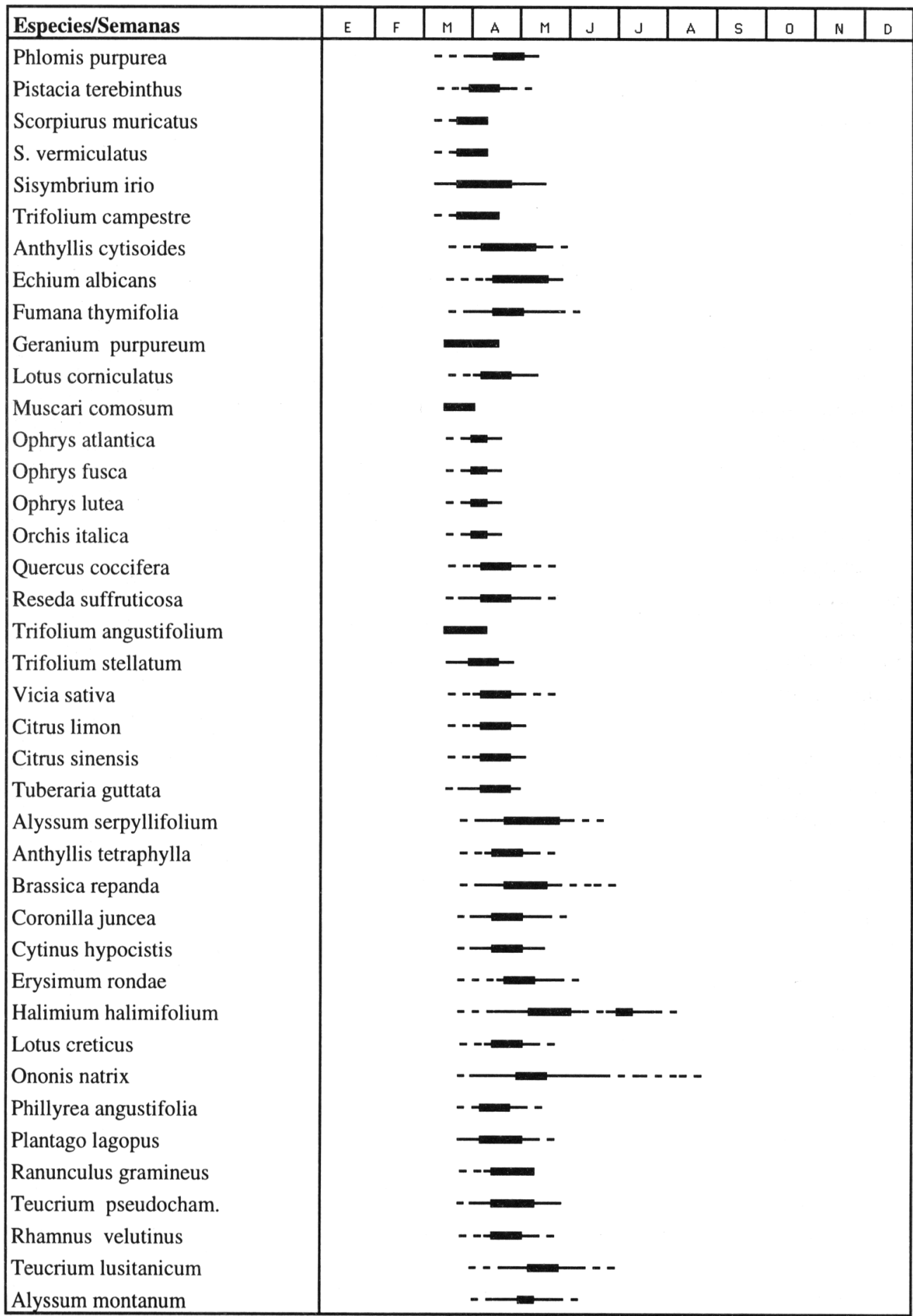




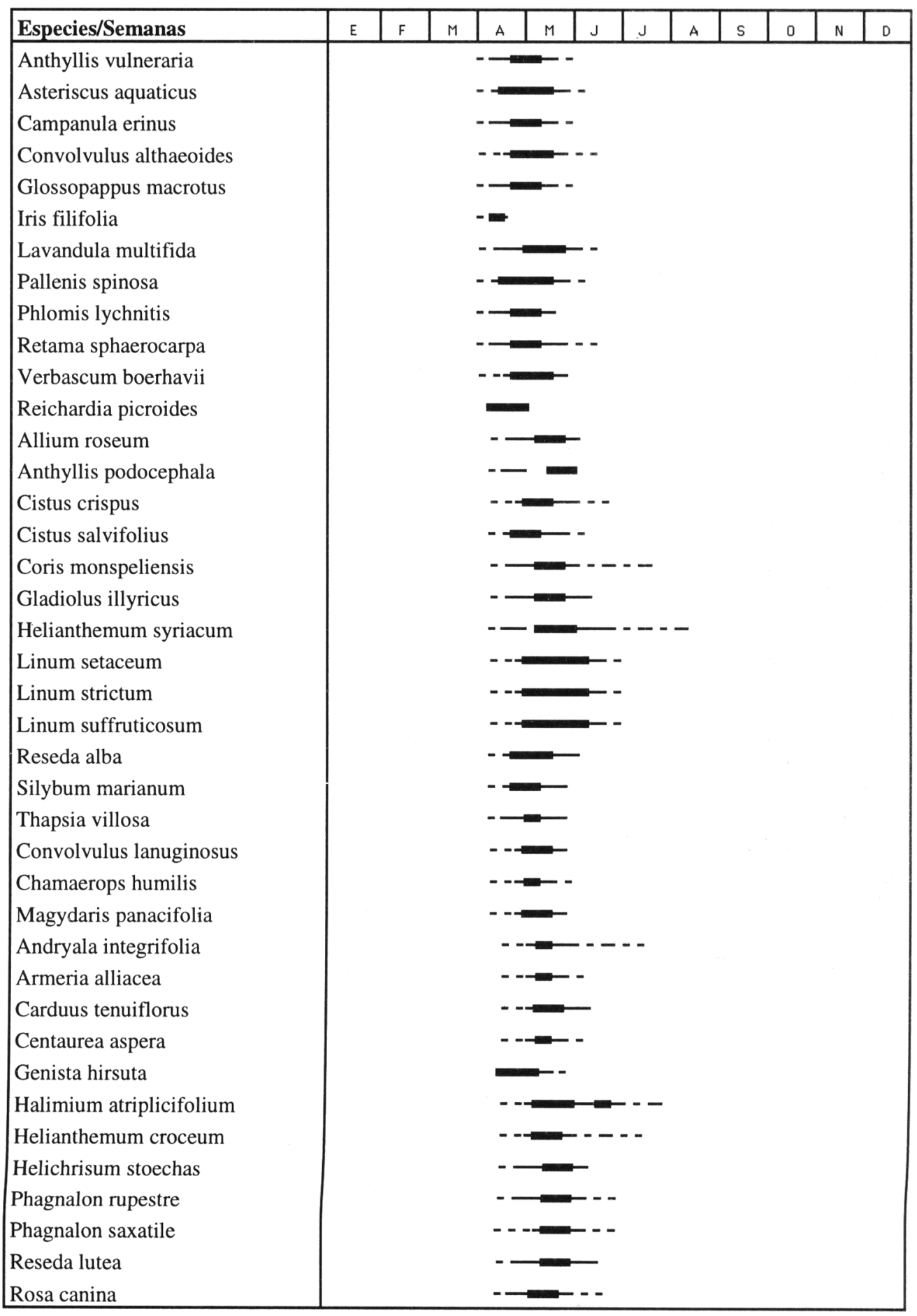




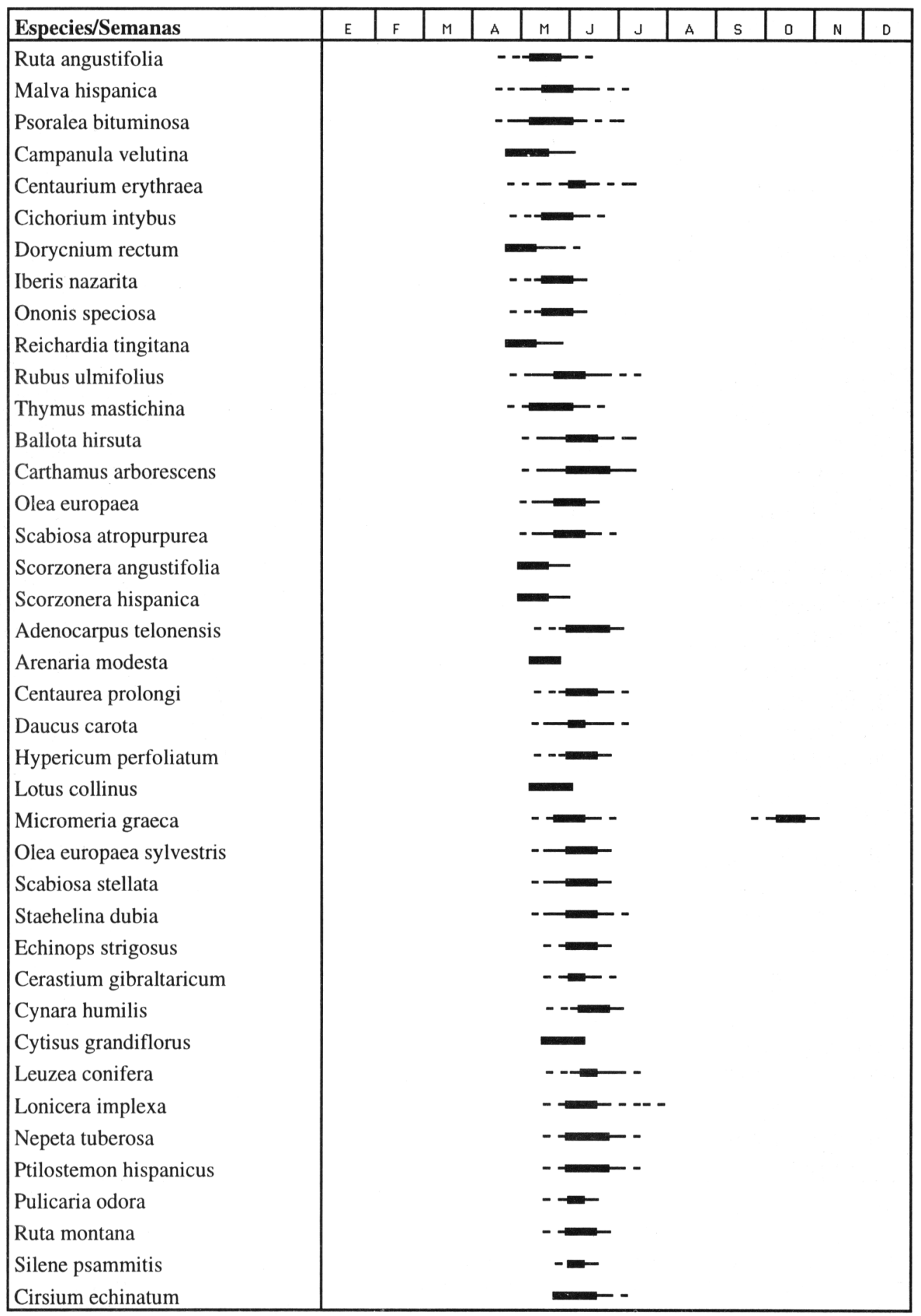




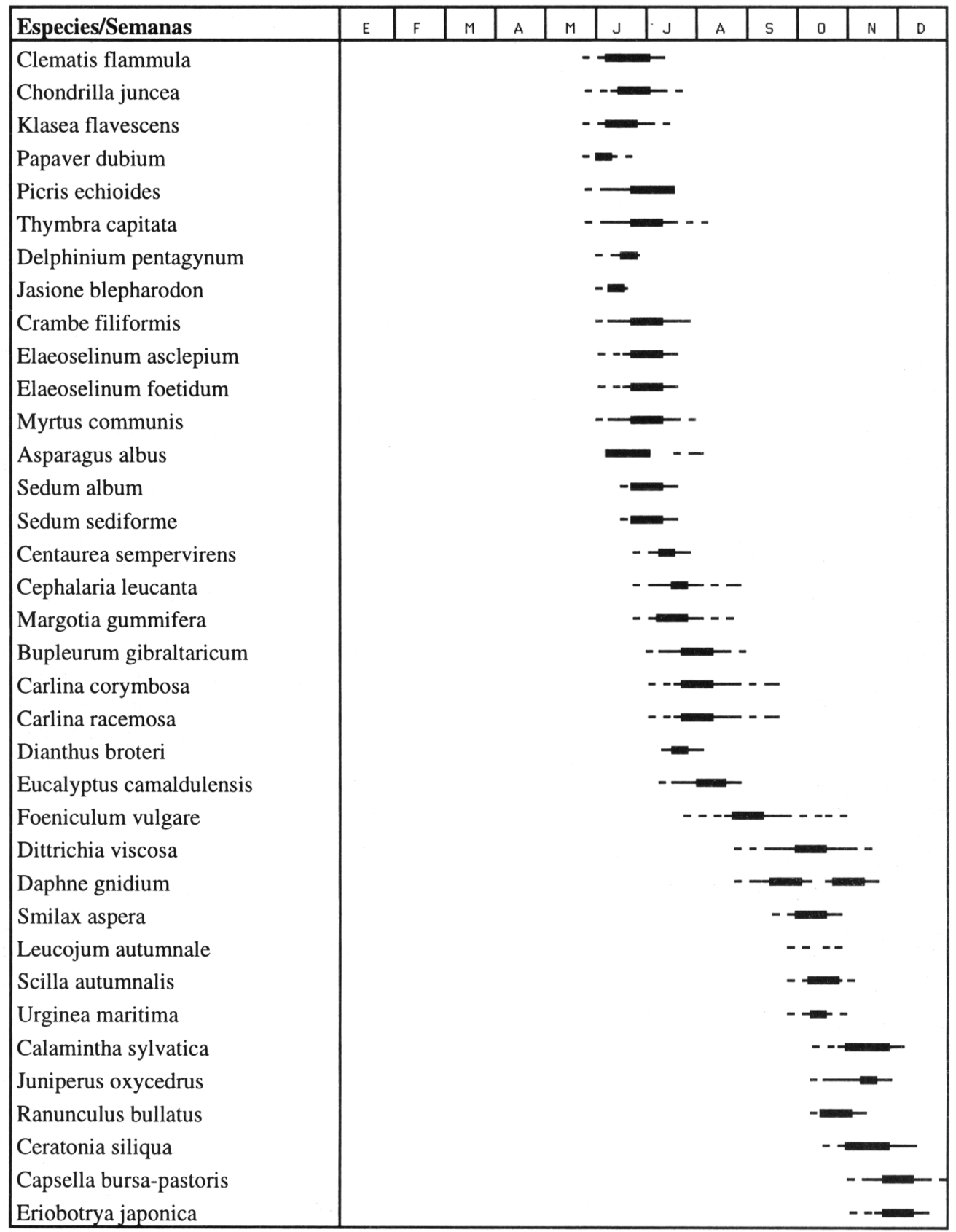

Tabla 3. Diagrama fenológico de las especies estudiadas en la Sierra de Mijas. Floración inicial y final (línea discontínua); floración pico (línea contínua). Phenologycal diagram of the species studied in Sierra de Mijas. Initial and final bloom periods (discontinous line). Full blooming period (continous line). 


\section{BIBLIOGRAFÍA}

ANDERSON, E. y L. HUBRICHT -1940- A method for describing and comparing blooming-season. Bull. Torrey Bot. Club, 67(8): 639-648.

ARROYO, J. -1983- Algunos aspectos fenológicos de la floración del matorral de Andalucía Occidental. En A. Blanco de Pablos (ed.) Avances sobre la investigación en Bioclimatología, 8: 263-272.

ARROYO, J. -1985- Fenología de la floración en matorrales de Andalucía Occidental. Tesis Doctoral. Universidad de Sevilla.

ARROYO, J. -1988b-. Fenología de la floración en especies del matorral del Sur de España. Lagascalia, 15(extra): 593-606.

ARROYO, J. -1990-. Ritmos climáticos y de floración en matorrales del SW de España. Lagascalia, 16(1): 25-50 .

ARROYO, J. -11988a-. Atributos florales y fenología de la floración en matorrales del Sur de España. Lagascalia, 15(1): 43-78.

BONET, A., J. RITA y M.T. SEBASTIA -1985-. $L a$ flora melífera de la circumscripció de Barcelona. Excma. Diputación de Barcelona.

CABEZUDO, B., A.V. PÉREZ LATORRE, T. NAVARRO y J.M. NIETO -1993- Estudios fenomorfológicos en la vegetación del sur de España. II. Alcornocales mesomediterráneos. (Montes de Málaga, España). Acta Bot. Malacitana, 18:179-188.

CABEZUDO, B., T. NAVARRO, A.V. PÉREZ LATORRE, J.M. NIETO y G. ORSHAN -1992Estudios fenomorfológicos en la vegetación del sur de España. I.Cistus. Acta Bot. Malacitana, 17: 229-237.

CANFIELD, R.H. -1941- Aplication of the "line intercept" method in sampling range vegetation. Jour. Four., 39: 388-394.

HERCE, P. -1942- Flora y regiones meliferas de España. Sección de publicaciones, prensa y propaganda del Ministerio de Agricultura. Madrid.

HERRERA, J. -1985a- Biología reproductiva del matorral de Doñana. Tesis Doctoral. Universidad de Sevilla.

HERRERA, J. - 1985b- Nectar secretion patterns in southern spanish mediterranean shrublands. Israel J. Bot., 34: 47-58.

HERRERA, J. -1986- Flowering and fruiting phenology in the coastal shrublands of Doñana, south Spain. Vegetatio, 68: 91-98.

HERRERA, J. -1987a- Biología reproductiva de algunas especies del matorral de Doñana. Anales Jard. Bot. Madrid, 44(2): 483-497.

HERRERA, J. -1987b- Flower and fruit biology en southern spanish mediterranean shrublands. Ann. Missouri Bot. Gard., 74: 69-78.

HERRERA, J. -1988a- Datos sobre biología floral en la flora de Andalucía Oriental. Lagascalia, 15(extra): 607-614.

HERRERA, J. -1988b- Pollination relationships in southern spanish mediterranean shrublands. $J$. Ecol., 76: 274-287.

HIDALGO, M.I., M.L. BOOTELLO y J. PACHECO -1990- Origen floral de las cargas de polen recogidas por Apis mellifera L. en Álora (Málaga, España). Acta Bot. Malacitana, 15: 33-44.

HILTY, S.L. -1980- Flowering and fruiting periodicity in a premontane rain forest in Pacific Colombia. Biotropica, 12: 292-306.

HOWES, F.N. -1953-Plantas melíferas. Reverté. Barcelona.

MARTÍN, J. y A. ESCARRE -1980- Datos de fenología, reducción de hoja y variaciones estacionales de la composición foliar, en cuatro especies del matorral costero del Mediterráneo meridional ibérico. Mediterránea, 4: 68-88.

NAVARRO, T., J.M. NIETO, A.V. PÉREZ LATORRE y B. CABEZUDO -1993- Estudios fenomorfológicos en la vegetación del sur de España. III. Comportamiento estacional de una comunidad de badlands (Tabernas, Almería. España). Acta Bot. Malacitana, 18: 189-198.

NIETO, J.M., A. PÉREZ LATORRE y B. CABEZUDO -1991- Biogeografía y series de vegetación de la provincia de Málaga (España). Acta Bot. Malacitana, 16(2): 417-436.

NIETO, J.M., S. PÉREZ y B. CABEZUDO -1987/ 88-. Datos sobre la vegetación dolomitícola del sector Rondeño (Sierra de Mijas, Málaga, España-. Lazaroa, 10: 35-46.

ORTEGA, J.L. -1987- Flora de interés apicola y polinización de los cultivos. Mundiprensa. Madrid.

ORTIZ, P. -1985- Análisis polínicos de las mieles y celdillas de las sierras del sur de Córdoba (España). An. Asoc. Palinol. Lengua Esp.: 2: 353-360.

ORTIZ, P. -1988- Estudio melitopalinológico en el 
Aldévalo (Huelva). Anales Asoc. Palinol. Lengua Esp.: 4: 64-72.

ORTIZ, P. -1990a- Aportación melitopalinológica al conocimiento de la flora apícola del norte de Córdoba. Lagascalia, 15(2): 165-177.

ORTIZ, P. -1990b- Contribución al conocimiento de la flora apícola gaditana. Lagascalia, 16(2): 199-210.

ORTIZ, P. -1991- Melitopalinología en Andalucía Occidental. Tesis Doctoral. Universidad de Sevilla.

ORTIZ, P., e I. FERNÁNDEZ -1992- Estudio microscópico de miel y polen apícola de la provincia de Sevilla. Acta Bot. Malacitana, 17: 183193.

ORTIZ, P., I FERNÁNDEZ y M. MARTÍN -1990Estudio melitopalinológico en la comarca de Aracena (Huelva). Lagascalia, 16(1): 61-76.

PÉREZ SANZ, S., J.M. NIETO y B. CABEZUDO 1987- Contribución al conocimiento de la flora de la sierra de Mijas (Málaga, España) Acta Bot. Malacitana, 12: 189-202.

RABIET, E. -1977- Choix et culture des plantes apicoles. Emile Rabiet. Saint-Martial de Vitaterne (Jonzac).

RICCIARDELLI, G. y L. PERSANO -1981-. Flora Apística Italiana. Instituto Sperimentale per la Zoología Agraria. Roma.

RITA, J. -1983- Flora melífera de la provincia de Lleida. Excma. Diputación de Lleida. Lleida.

SAURY, A. -1981-Les plantes mellifères. Lechevalier. Paris.
SERRA, J. -1988- Origen botánico del polen producido en España. Anales Asoc. Palinol. Lengua Esp., 4: 73-78.

SOCORRO, O. - 1985- Flora melífera. En M. Ferrer (eds) Sierra Nevada y la Apujarra, IV: 13771405.

TALAVERA, S., J. HERRERA, J. ARROYO, P.L. ORTIZ y J.A. DEVESA -1988- Estudio de la flora apícola de Andalucía Occidental. Lagascalia, 15 (extra): 567-591.

Aceptado para su publicación en Junio de 1994

Dirección de los autores. Dpto. Biología Vegetal. Facultad de Ciencias. Universidad de Málaga. Apdo. 59. 29080, Málaga, Fax. (95) 2131944. 